\title{
On Algebraic Spectrum of Ontology Evaluation
}

\author{
Adekoya Adebayo Felix ${ }^{1}$, Akinwale Adio Taofiki ${ }^{2}$ \\ Department of Computer Science, \\ University of Agriculture, \\ Abeokuta, Nigeria
}

\begin{abstract}
Ontology evaluation remains an important open problem in the area of its application. The ontology structure evaluation framework for benchmarking the internal graph structures was proposed. The framework was used in transport and biochemical ontology. The corresponding adjacency, incidence matrices and other structural properties due to the class hierarchical structure of the transport and biochemical ontology were computed using MATLAB. The results showed that the choice of suitable choice must depend on the purpose of ontology structure evaluation.
\end{abstract}

Keywords- Ontology evaluation; adjacency matrix; graph; algebraic spectrum.

\section{INTRODUCTION}

Class relationships are represented by three special kinds of graphs; namely lattice, tree and acyclic, which supports partial ordering Sowa (2004). An ontology describes class relationships using acyclic graphs and provides a convenient means of using appropriate tools to analyze and solve a given concepts. Ontology uses two graph components: nodes and edges to represents concepts (classes, objects, and entities), and the relations between these concepts respectively. In practice, ontologies are acyclic graph models which describe the semantic relationships between the inherent concepts and relations, between concepts and concepts, and between relations and relations. The internal structure and the structural dimension reveal their computational complexity and memory utilization, and also aid fast concept-relation retrievals.

A subset of the nodes and edges in a graph possesses certain characteristics or relate to each other in particular ways which are quite useful for describing the structural relationships existing among concepts, relations and between concepts and relations in ontology. A vast amount of ontologies for diverse domain of discourse have been designed and are available across many ontology libraries. Thus many applications in such areas as knowledge engineering, artificial intelligence, natural language processing, e-commerce, database design and integration, bioinformatics, semantic web, education have been developed using these ontologies. However, many of these ontologies are not well documented and evaluated, therefore hindering their effective re-use and inter-operability in many applications (Staab, 2009 and Lu and Haarslev, 2006).

The lack of tools to analyze these ontologies has not helped the effective utilization of the inherent knowledge that is contained in these ontologies. In this paper, an algebraic framework for benchmarking the internal graph structures of ontologies was proposed.

\author{
Sofoluwe Adetokunbo ${ }^{3}$ \\ Department of Computer Science, \\ University of Lagos, \\ Lagos, Nigeria
}

\section{LITERATURE REVIEWS}

There is a need to evaluate the ontology and also the results from the ontology. Many researchers have tried various ways of evaluation of ontologies. Maedche and Staab (2002) proposed the evaluation of ontology using lexical or vocabulary level. The process compared two strings based on the Levenshtein edit distance and it was normalized to produce scores in the range of 0 and 1. In 2005, Velardi et al used a body of natural language text to generate natural language glosses for multiple word terms. Part of their techniques employed is-a relation. Brewster et al (2004) suggested using a data-driven approach to evaluate the degree of structural fit between an ontology and a corpus of documents. The ideas was based on clustering algorithm using unsupervised way. Spyns (2005) used relational model to evaluate ontology by extracting a set of lexons from natural language text. Ding et al (2004) used cross-reference between semantic web documents to define a graph and then compute a score for each ontology. Porzel and Malaka (2004) described a scenario where the ontology with its relations was used primarily to determine how closely related the meaning of two concepts was by using the final output of speech recognition problem as a case study.

Patel et al (2005) showed how to determine if the ontology was referred to a particular topic and classified the ontology into a directory topics using standard machine learning algorithms. Brewster et al (2004) extracted a set of relevant domain specific terms from the corpus of documents using latent semantic analysis. Bruton-Jones et al (2004) proposed ten simple criteria of lawfulness, richness, interpretability, consistency, clarity, comprehensiveness, accuracy, relevant, authority and history to evaluate the ontology which were based on scores. Fox et al (1998) proposed another set of criteria which were geared more towards manual assessment and evaluation of ontologies. Luzano-Tello and Gomez-Perez (2004) defined an even more detailed sets of 117 criteria, organized in a three- level framework for evaluation of ontology. Willen Rober et al (2005) proposed two alternative techniques for the evaluation of ontology-matching systems. In this work structural dimensions of ontology by Gangen et al (2006) were used to evaluate the ontology and characteristic polynomial equation of eigenspace with their corresponding adjacency matrix.

\section{SPECTRAL ANALYSIS OF GRAPHS}

Graph spectrum is the set of its eigenvalues in addition with their multiplicities. There are two standards computational representations of graphs; namely as a collection of adjacency lists or as an adjacency matrix. The adjacency-list representation provides compact way to represent sparse graphs 
where $\left(|E|<|V|^{2}\right)$. The adjacency-matrix of a graph $G=(V, E)$ consists of a $|V| \mathrm{x}|V|$ matrix $A=\left(a_{i j}\right)$

such that $a_{i j}= \begin{cases}1 & \text { if }(i, j) \in E \\ 0 & \text { otherwise }\end{cases}$

where the vertices are numbered $1,2,3, \ldots,|V|$ in some arbitrary order. The adjacency-matrix $A$ of an undirected graph is its own transpose

$$
\text { i.e } A=A^{T} \text {, If } A=\left(a_{i j}\right) \text { then } A^{T}=\left(a_{i j}{ }^{T}\right) \text { and } a_{i j}{ }^{T}=a_{j i} \text {. }
$$

In undirected graph, $(u, v)=(v, u)$ means the same edge. In some applications, it pays to store only the entries on and above the diagonal of the adjacency matrix, therefore cutting the memory needed to store the graph almost in half.

The simplicity of an adjacency-matrix makes it preferable to compute the algebraic spectrum especially with small graphs. For weighted graph, there is an additional advantage in the sense that the adjacency matrix uses only one bit per entry instead of one word of computer memory for each matrix entry. Adjacency-matrix makes it easier to search for sub-graphs of particular pattern or identities.

The incidence matrix for an undirected graph $G=(V, E)$ is a $|V| \mathrm{x}|E|$ matrix $M$

such that

$$
M_{v e}=\left\{\begin{array}{l}
1 \text { if edge } e \text { is incident on vertex } v \\
0 \text { otherwise }
\end{array}\right.
$$

A set of columns of $M$ is linearly independent if and only if the corresponding set of edges is acyclic.

The incidence matrix for a digraph $G=(V, E)$ is a $|V| \mathrm{x}|E|$ is a matrix $M$ such that

$$
M_{v e}=\left\{\begin{array}{cl}
-1 & \text { f edge e leaves vertex } v \\
1 & \text { if edge e enters vertex } v \\
0 & \text { otherwise }
\end{array}\right.
$$

If a set of edges in $G$ is linearly independent, then the corresponding set of edges does not contain a directed cycle.

Whereas the adjacency-matrix encapsulate vertex-to-vertex relationships, the incidence matrix encapsulate vertex-to-edge relationships and its Eigen values reveals the relationship between two classes of objects. The advantage of the incidence matrix is that it enables data to be rapidly indexed either for vertices or for edges.

\section{An Illustration of SPECTRAL Analysis of ONTOLOGICAL STRUCTURE}

Ontologies are directed acyclic graphs and since an inverse of a relation can be obtained from that relation, it follows that information flows in both direction. Consider the transport ontology in figure 1, we computed the adjacency and incidence matrices as depicted tables $1-4$.
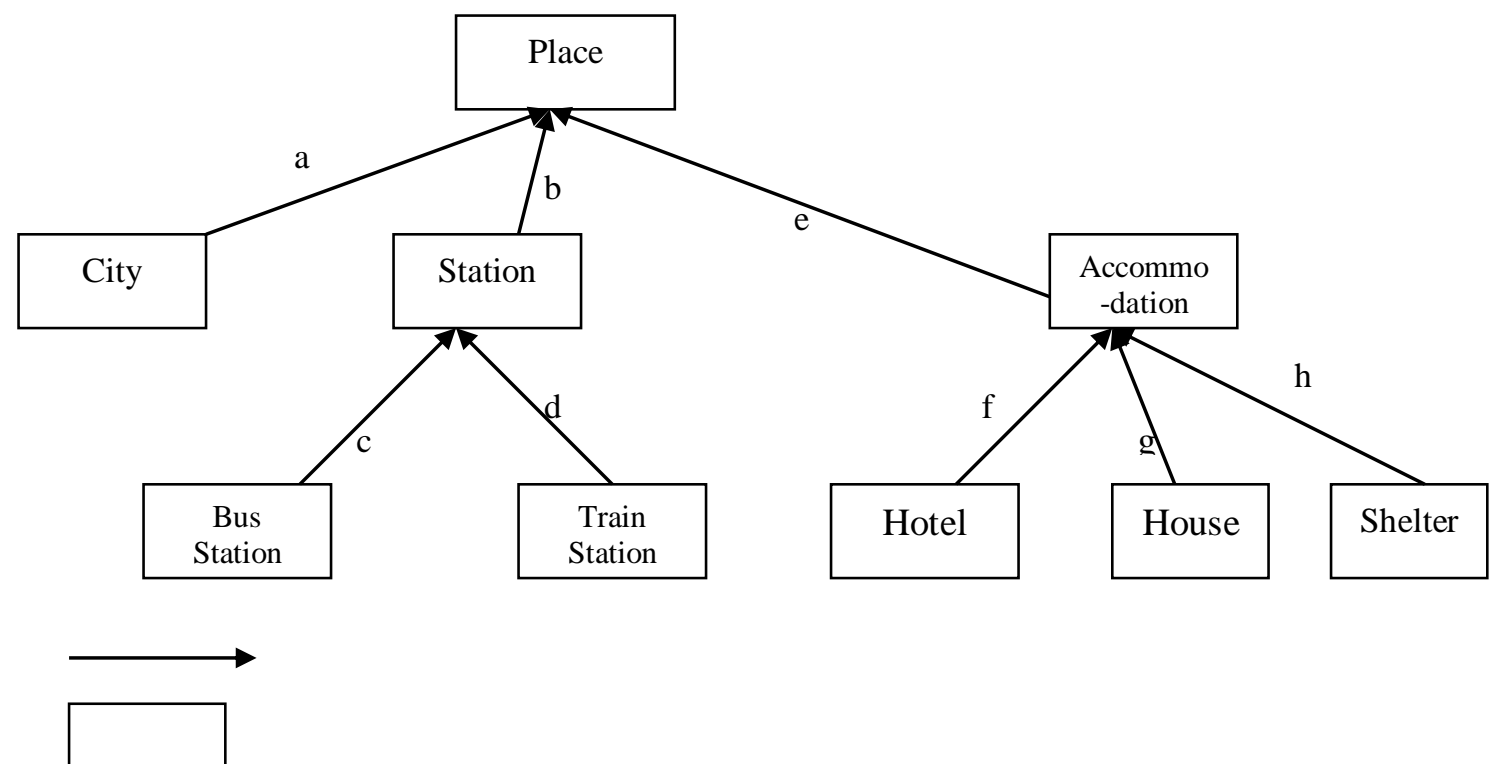

Figure 1: Transport ontology with $V=\{$ Place, City, Station, Bus Station, Train Station, Accommodation, Hotel, House, Shelter $\}$ and $E=\{a, b, c, d, e, f, g, h\}$

TABLE 1: Incidence matrix of DAG ontological Structure in Figure 1

\begin{tabular}{|l|c|c|c|c|c|c|c|c|}
\hline & a & B & C & d & E & f & g & h \\
\hline Place & 1 & 1 & 0 & 0 & 1 & 0 & 0 & 0 \\
\hline City & -1 & 0 & 0 & 0 & 0 & 0 & 0 & 0 \\
\hline Station & 0 & -1 & 1 & 1 & 0 & 0 & 0 & 0 \\
\hline Bus Station & 0 & 0 & -1 & 0 & 0 & 0 & 0 & 0 \\
\hline Train Station & 0 & 0 & 0 & -1 & 0 & 0 & 0 & 0 \\
\hline Accommodation & 0 & 0 & 0 & 0 & -1 & 1 & 1 & 1 \\
\hline
\end{tabular}




\begin{tabular}{|l|l|l|l|l|l|l|l|l|}
\hline Hotel & 0 & 0 & 0 & 0 & 0 & -1 & 0 & 0 \\
\hline House & 0 & 0 & 0 & 0 & 0 & 0 & -1 & 0 \\
\hline Shelter & 0 & 0 & 0 & 0 & 0 & 0 & 0 & -1 \\
\hline
\end{tabular}

TABLE 2: Incidence matrix of undirected ontological structure of Figure 1 (Inverse relation)

\begin{tabular}{|l|l|l|l|l|l|l|l|l|}
\hline & A & b & c & d & E & f & g & H \\
\hline Place & 1 & 1 & 0 & 0 & 1 & 0 & 0 & 0 \\
\hline City & 1 & 0 & 0 & 0 & 0 & 0 & 0 & 0 \\
\hline Station & 0 & 1 & 1 & 1 & 0 & 0 & 0 & 0 \\
\hline Bus Station & 0 & 0 & 1 & 0 & 0 & 0 & 0 & 0 \\
\hline Train Station & 0 & 0 & 0 & 1 & 0 & 0 & 0 & 0 \\
\hline Accommodation & 0 & 0 & 0 & 0 & 1 & 1 & 1 & 1 \\
\hline Hotel & 0 & 0 & 0 & 0 & 0 & 1 & 0 & 0 \\
\hline House & 0 & 0 & 0 & 0 & 0 & 0 & 1 & 0 \\
\hline Shelter & 0 & 0 & 0 & 0 & 0 & 0 & 0 & 1 \\
\hline
\end{tabular}

TABLE 3: Adjacency matrix of DAG ontological Structure

\begin{tabular}{|l|c|c|c|c|c|c|c|c|c|}
\hline & Place & City & Station & Bus Station & Train Station & $\begin{array}{c}\text { Accomm- } \\
\text { odation }\end{array}$ & Hotel & House & Shelter \\
\hline Place & 0 & 0 & 0 & 0 & 0 & 0 & 0 & 0 & 0 \\
\hline City & 1 & 0 & 0 & 0 & 0 & 0 & 0 & 0 & 0 \\
\hline Station & 1 & 0 & 0 & 0 & 0 & 0 & 0 & 0 & 0 \\
\hline Bus Station & 0 & 0 & 1 & 0 & 0 & 0 & 0 & 0 & 0 \\
\hline Train Station & 0 & 0 & 1 & 0 & 0 & 0 & 0 & 0 & 0 \\
\hline Accommodation & 1 & 0 & 0 & 0 & 0 & 0 & 0 & 0 & 0 \\
\hline Hotel & 0 & 0 & 0 & 0 & 0 & 1 & 0 & 0 & 0 \\
\hline House & 0 & 0 & 0 & 0 & 0 & 1 & 0 & 0 & 0 \\
\hline Shelter & 0 & 0 & 0 & 0 & 0 & 1 & 0 & 0 & 0 \\
\hline
\end{tabular}

TABLE 4: Adjacency-matrix of the undirected ontological structure

\begin{tabular}{|l|c|c|c|c|c|c|c|c|c|}
\hline & Place & City & Station & Bus Station & Train Station & $\begin{array}{c}\text { Accomm- } \\
\text { odation }\end{array}$ & Hotel & House & Shelter \\
\hline Place & 0 & 1 & 1 & 0 & 0 & 1 & 0 & 0 & 0 \\
\hline City & 1 & 0 & 0 & 0 & 0 & 0 & 0 & 0 & 0 \\
\hline Station & 1 & 0 & 0 & 1 & 1 & 0 & 0 & 0 & 0 \\
\hline Bus Station & 0 & 0 & 1 & 0 & 0 & 0 & 0 & 0 & 0 \\
\hline Train Station & 0 & 0 & 1 & 0 & 0 & 0 & 0 & 0 & 0 \\
\hline Accommodation & 1 & 0 & 0 & 0 & 0 & 0 & 1 & 1 & 1 \\
\hline Hotel & 0 & 0 & 0 & 0 & 0 & 1 & 0 & 0 & 1 \\
\hline House & 0 & 0 & 0 & 0 & 0 & 1 & 1 & 0 & 0 \\
\hline Shelter & 0 & 0 & 0 & 0 & 0 & 1 & 0 & 0 & 0 \\
\hline
\end{tabular}

\section{Computing Eigen Values And Eigen Vectors}

In this work, we used the characteristic polynomial equation to compute the eigenspace of the example ontology given in figure 1 and the corresponding adjacency matrix in Table 4.

If $A$ is an $n \times n$ matrix, then its corresponding eigenvlues $(\lambda)$ are the roots of the characteristics polynomial
$\operatorname{det}(A-\lambda I)$ and the eigenvectors would be $v_{1}, v_{2}, v_{3}, \ldots, v_{n}$ respectively. An eigenvalue $\lambda$ mapped to an eigenvector and the set of all eigenvectors which satisfy $A v=\lambda v$ are referred to as the eigenspace of $A . v$ must be a non-zero and the characteristics polynomial must be set to zero.

$$
\text { i.e } \operatorname{det}(A-\lambda I)=0
$$


The number of eigenvalues for a $n \times n$ matrix is $n$, though some of the eigenvalues might be equal to one another.
Consider the ontology figure 1 and the adjacency matrix in table 4, the eigenvalues are

Transont $(9 \times 9)=$

$$
\left[\begin{array}{lllllllll}
0 & 1 & 1 & 0 & 0 & 1 & 0 & 0 & 0 \\
1 & 0 & 0 & 0 & 0 & 0 & 0 & 0 & 0 \\
1 & 0 & 0 & 1 & 1 & 0 & 0 & 0 & 0 \\
0 & 0 & 1 & 0 & 0 & 0 & 0 & 0 & 0 \\
0 & 0 & 1 & 0 & 0 & 0 & 0 & 0 & 0 \\
1 & 0 & 0 & 0 & 0 & 0 & 1 & 1 & 1 \\
0 & 0 & 0 & 0 & 0 & 1 & 0 & 0 & 1 \\
0 & 0 & 0 & 0 & 0 & 1 & 1 & 0 & 0 \\
0 & 0 & 0 & 0 & 0 & 1 & 0 & 0 & 0
\end{array}\right]
$$

The eigenvalues $\operatorname{det}(A-\lambda \boldsymbol{I})=\left[\begin{array}{ccccccccc}-\lambda & 1 & 1 & 0 & 0 & 1 & 0 & 0 & 0 \\ 1 & -\lambda & 0 & 0 & 0 & 0 & 0 & 0 & 0 \\ 1 & 0 & -\lambda & 1 & 1 & 0 & 0 & 0 & 0 \\ 0 & 0 & 1 & -\lambda & 0 & 0 & 0 & 0 & 0 \\ 0 & 0 & 1 & 0 & -\lambda & 0 & 0 & 0 & 0 \\ 1 & 0 & 0 & 0 & 0 & -\lambda & 1 & 1 & 1 \\ 0 & 0 & 0 & 0 & 0 & 1 & -\lambda & 0 & 1 \\ 0 & 0 & 0 & 0 & 0 & 1 & 1 & -\lambda & 0 \\ 0 & 0 & 0 & 0 & 0 & 1 & 0 & 0 & -\lambda\end{array}\right]$

We use MATLAB to compute the $\operatorname{det}(A-\lambda I)$ since $n$ is large.

Therefore, $\operatorname{det}(A-\lambda I)=\lambda^{9}-8 \lambda^{7}-2 \lambda^{6}+15 \lambda^{5}+8 \lambda^{4}-2 \lambda^{3}-4 \lambda^{2}-2 \lambda$

The eigenvalues are 2.4046, 1.6785, $-2.1667,0.7390,-1.4299$

$-0.6731,-0.2762+0.4952 \mathrm{i},-0.2762-0.4952 \mathrm{i}$ and 0.0000 .

The eigenvectors $(\mathrm{V})=$

$\left[\begin{array}{cccccc}-0.4345 & 0.5532 & 0.2891 & -0.0162 & -0.4694 & -0.4190 \\ -0.1807 & -0.2553 & 0.1722 & 0.0113 & -0.6351 & 0.6225 \\ -0.2762 & -0.4448 & 0.5936 & 0.5174 & 0.2386 & -0.1823 \\ -0.1149 & 0.2053 & 0.3537 & -0.3619 & 0.3229 & 0.2708 \\ -0.1149 & 0.2053 & 0.3537 & -0.3619 & 0.3229 & 0.2708 \\ -0.5878 & -0.4985 & -0.2806 & -0.5056 & 0.0496 & -0.1582 \\ -0.3461 & 0.1239 & -0.2668 & 0.1063 & 0.1580 & -0.1141 \\ -0.3884 & 0.1729 & -0.3261 & 0.2793 & 0.2809 & 0.4046 \\ -0.2444 & 0.2301 & -0.1672 & 0.3536 & 0.0671 & 0.2350\end{array}\right]$

$\mathrm{D}=\left[\begin{array}{rrrrrrrr}2.4046 & 0 & 0 & 0 & 0 & 0 & \\ 0 & -2.1667 & 0 & 0 & 0 & 0 \\ 0 & 0 & 1.6785 & 0 & 0 & 0 \\ 0 & 0 & 0 & -1.4299 & 0 & 0 \\ 0 & 0 & 0 & 0 & 0.7390 & 0 \\ & 0 & 0 & 0 & 0 & 0 & -0.6731\end{array}\right]$


where $\mathrm{V}$ represents the set of eigenvectors and $\mathrm{D}$ the set of of eigenvalues. The flag $=0$ implies that the eigenvalues converged.

\section{StruCtural Dimension Metrics OF GRAPHS}

The structural dimension of ontologies focuses on analysis of digraph structures and formal semantics. Gangemi, Catenacci, Ciaramita and Lehmann (2006) proposed the following formula which were used in the proposed ONTOSTEVAL for measuring the structural dimensions of ontologies among others. We compute the structural dimension of the sample ontology in figure 1.

\section{A. Measures for depth}

This measure is related to the cardinality of paths in a digraph where the arcs are only is- $a$ relations. Three types of depth measures namely; absolute depth, average depth and maximal depth were used in the proposed ONTOSTEVAL.

1) Absolute Depth

$$
D_{a b s}=\sum_{j}^{P} N_{j \in P}
$$

where $N_{j \in P}$ is the cardinality of each path $j$ from the set of paths $P$ in a graph $G$.

From figure 1,

$$
\begin{gathered}
P=\{\mathrm{a}, \mathrm{bc}, \mathrm{bd}, \mathrm{ef}, \mathrm{eg}, \mathrm{eh}\}=6 \text { paths } \\
\left.\qquad \begin{array}{l}
\{\mathrm{a}\} \\
\{\{\mathrm{b}\},\{\mathrm{c}\}\} \\
\{\{\mathrm{b}\},\{\mathrm{d}\}\} \\
\{\{\mathrm{e}\},\{\mathrm{f}\}\} \\
\{\{\mathrm{e}\},\{\mathrm{g}\}\} \\
\{\{\mathrm{e}\},\{\mathrm{h}\}\}
\end{array}\right\}=\left\{\begin{array}{l}
1 \\
2 \\
2 \\
2 \\
2 \\
2
\end{array}\right\}, \\
\text { therefore } D_{a b s}=11 .
\end{gathered}
$$

2) Average Depth

$$
D_{\text {ave }}=\frac{1}{n_{p \subseteq G}} \sum_{j}^{P} N_{j \in P}
$$

where $N_{j \in P}$ is the cardinality of each path $j$ from the set of paths $P$ in a graph $G$, and $n_{p \subseteq G}$ is the cardinality of $P$.

3) Maximal Depth

$$
D_{\text {ave }}=\frac{1}{6}(11)=1.83
$$

$$
\begin{aligned}
& D_{m}=N_{j \in P} \\
& \forall i \exists j\left(N_{j \in P} \geq N_{i \in P}\right.
\end{aligned}
$$

where $N_{j \in P}$ and $N_{i \in P}$ are the cardinalities of any path $i$ or $j$ from the set of paths $P$ in a graph $G$.

$$
D_{m}=2 \text {. }
$$

\section{B. Measures for breadth}

This measure is related to the cardinality of generations in a graph where the edges are only the $i s$ - $a$ relations. Three types of depth measures namely; absolute depth, average depth and maximal depth were used in the proposed ONTOSTEVAL.

1) Absolute Breadth

$$
B_{a b s}=\sum_{j}^{G_{L}} N_{j \in G_{L}}
$$

where $N_{j \in G_{L}}$ is the cardinality of each generation $j$ from the set of generations $G_{L}$ in the digraph $G$.

There are three levels $\left(G_{L}\right)$ in the example ontology in figure 1 namely

Level $1=\{1\}$ contain 1 vertex

Level $2=\{2,3,4\}$ contain 3 vertexes

Level $3=\{5,6,7,8,9\}$ contain 5 vertexes

Therefore, $\quad B_{a b s}=9$

2) Average Breadth

$$
B_{\text {ave }}=\frac{1}{n_{G_{L} \subseteq G}} \sum_{j}^{G_{L}} N_{j \in G_{L}}
$$

where $N_{j \in G_{L}}$ is the cardinality of each generation $j$ from the set of generations $G_{L}$ in a digraph $G$, and $n_{G_{L} \subseteq G}$ is the cardinality of $G_{L}$.

$$
B_{\text {ave }}=\frac{1}{3}(9)=3
$$

3) Maximal breadth

$$
\begin{aligned}
& B_{m}=N_{j \in G_{L}} \\
& \forall i \exists j\left(N_{j \in G_{L}} \geq N_{i \in G_{L}}\right)
\end{aligned}
$$

where $N_{j \in G_{L}}$ and $N_{i \in G_{L}}$ are the cardinalities of any generation $i$ or $j$ from the set of generations $G_{L}$ in a graph $G$.

$$
B_{m}=5 \text { (the highest number of vertexes) }
$$

\section{Measures for density}

Gangemi (2005) defined density as the presence of clusters of classes with many non-taxonomical relations holding among them. We defined density based Coleman \& Moré (1983) on as follows 


$$
D=\frac{2|E|}{|V|(|V|-1)}
$$

where $|E|$ is total number of edges and $|V|$ is the total number of vertexes. The maximum number of edges is $1 / 2|V|$ $(|V|-1)$, so the maximal density is 1 (for complete graphs) and the minimal density is 0 .

$$
\begin{aligned}
& \mid \begin{array}{l}
E \mid=\{\mathrm{a}, \mathrm{b}, \mathrm{c}, \mathrm{d}, \mathrm{e}, \mathrm{f}, \mathrm{g}, \mathrm{h}\}=8 \\
V \mid=\{1,2,3,4,5,6,7,8,9\}=9
\end{array} \\
& D=\frac{2 \times 8}{9(9-1)}=0.22
\end{aligned}
$$

\section{ONTOlOGy STRUCTURE EVALUATION (ONTOSTEVAL) FRAMEWORK}

The proposed Ontology Structure Evaluation (ONTOSTEVAL) Framework for benchmarking the internal graph structures of ontologies is described in this section. The ONTOSTEVAL architecture is presented in figure 2 and comprises of three sections, namely the input, processing and the output units. The input unit accepts an ontology written in OWL such as the domain ontology while the processing unit parses the OWL file, analyses and computes its internal structures such as the spectral properties.

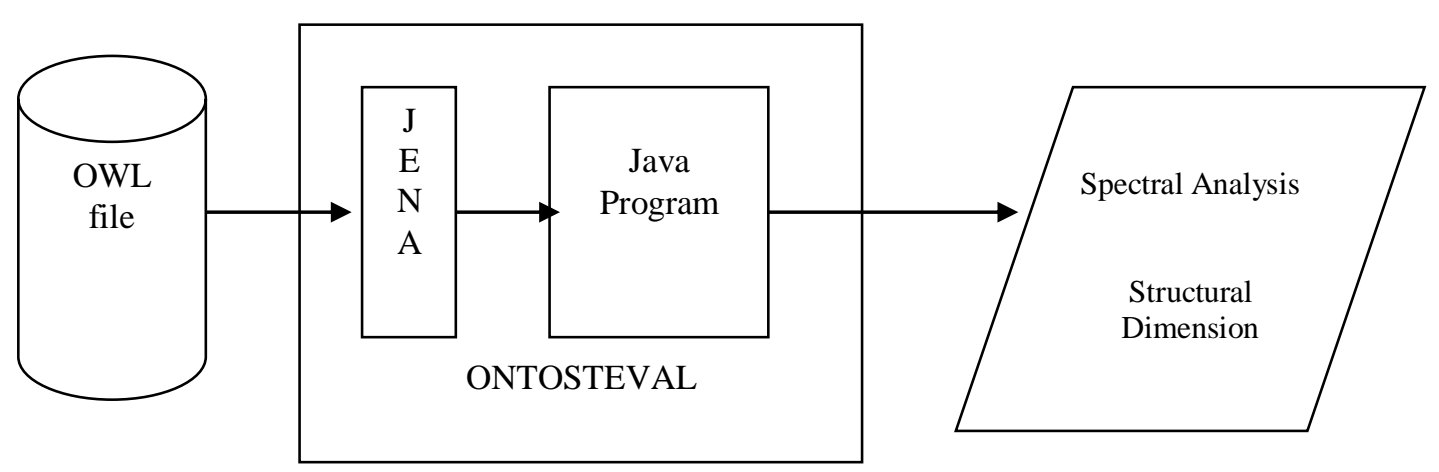

Figure 2: The ONTOSTEVAL Architecture

The Java program is embedded into Jena Framework and uses the well-known depth-first and breadth-first algorithms to determine the adjacent vertexes and the edges incident on a vertex as illustrated in figure 3. A Java program was written to compute the spectral analysis (adjacency and incidence matrices, eigenvalues and eigenvectors) and the structural dimension (depth, breadth and density) for ontologies written in OWL. The Java program was embedded in Jena Semantic Web Framework in other to enable the Java program read, parse and analyze any ontology file, and computes the inherent spectral and structural dimension metrics. In this section we present the spectral and structural analyses of some ontologies which were used with the proposed Ontology Structural Evaluation Framework (ONTOSTEVAL).

Biochemical Ontology

Biochemical ontology aim to capture and represent biochemical entities and the relations that exist between them in an accurate and precise manner. Figures 3 described the class hierarchical structure of the biochemical ontology while figure 4 shows its graph in the protégé implementation.

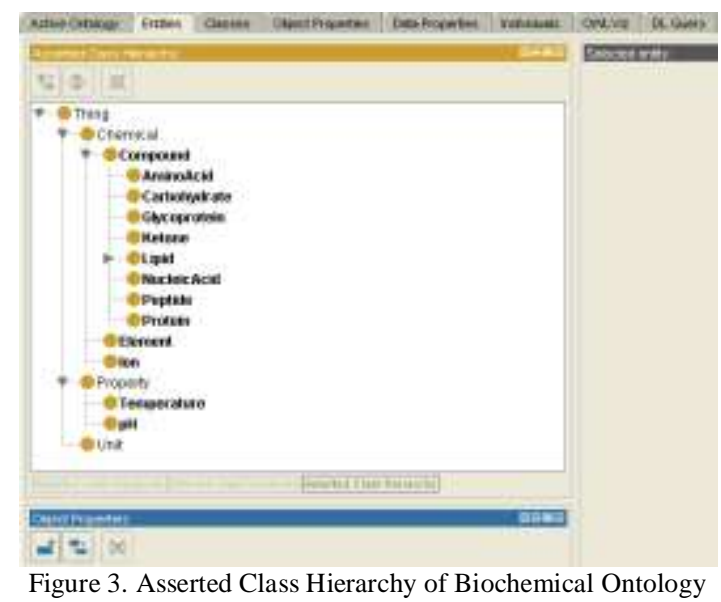

www.ijacsa.thesai.org 


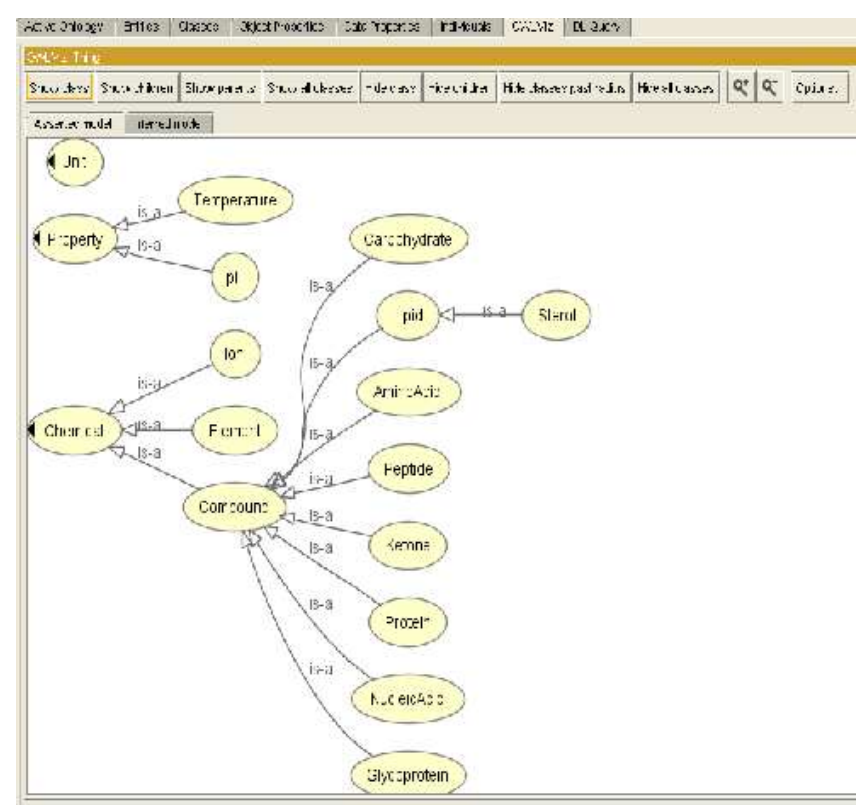

Figure 4. Biochemical OWL graph

The spectral and structural dimension properties which were subsequently computed using MATLAB are as follows:

Adjacency matrix of the Biochemical ontology =

$\left[\begin{array}{lllllllllllll}0 & 1 & 1 & 1 & 0 & 0 & 0 & 0 & 0 & 0 & 0 & 0 & 0 \\ 1 & 0 & 0 & 0 & 0 & 0 & 0 & 0 & 0 & 0 & 0 & 0 & 0 \\ 1 & 0 & 0 & 0 & 0 & 0 & 0 & 0 & 0 & 0 & 0 & 0 & 0 \\ 1 & 0 & 0 & 0 & 1 & 1 & 1 & 1 & 0 & 1 & 1 & 1 & 1 \\ 0 & 0 & 0 & 1 & 0 & 0 & 0 & 0 & 0 & 0 & 0 & 0 & 0 \\ 0 & 0 & 0 & 1 & 0 & 0 & 0 & 0 & 0 & 0 & 0 & 0 & 0 \\ 0 & 0 & 0 & 1 & 0 & 0 & 0 & 0 & 0 & 0 & 0 & 0 & 0 \\ 0 & 0 & 0 & 1 & 0 & 0 & 0 & 0 & 1 & 0 & 0 & 0 & 0 \\ 0 & 0 & 0 & 0 & 0 & 0 & 0 & 1 & 0 & 0 & 0 & 0 & 0 \\ 0 & 0 & 0 & 1 & 0 & 0 & 0 & 0 & 0 & 0 & 0 & 0 & 0 \\ 0 & 0 & 0 & 1 & 0 & 0 & 0 & 0 & 0 & 0 & 0 & 0 & 0 \\ 0 & 0 & 0 & 1 & 0 & 0 & 0 & 0 & 0 & 0 & 0 & 0 & 0 \\ 0 & 0 & 0 & 1 & 0 & 0 & 0 & 0 & 0 & 0 & 0 & 0 & 0\end{array}\right]$

The characteristics polynomial, det $(A-\lambda I)=\lambda^{13}-12 \lambda^{11}+26 \lambda^{9}-14 \lambda^{7}$

Eigenvalues $=-3.0643,-1.3288,-0.9189,-0.0000,-0.0000,-0.0000,0.0000,0.0000$,

$0.0000,0.0000,0.9189,1.3288,3.0643$.

The Eigenvectors $(\mathrm{V})=$

$\left[\begin{array}{ccccccc}-0.2860 & -0.6411 & 0.0850 & 0.0850 & -0.6411 & -0.2860 \\ -0.0933 & -0.4824 & 0.0925 & -0.0925 & 0.4824 & 0.0933 \\ -0.0933 & -0.4824 & 0.0925 & -0.0925 & 0.4824 & 0.0933 \\ -0.6898 & 0.1130 & -0.1069 & 0.1069 & -0.1130 & 0.6898 \\ -0.2251 & 0.0850 & -0.1163 & -0.1163 & 0.0850 & -0.2251 \\ -0.2251 & 0.0850 & -0.1163 & -0.1163 & 0.0850 & -0.2251 \\ -0.2251 & 0.0850 & -0.1163 & -0.1163 & 0.0850 & -0.2251 \\ -0.2519 & 0.1960 & 0.6309 & 0.6309 & 0.1960 & -0.2519 \\ -0.0822 & 0.1475 & 0.6866 & -0.6866 & -0.1475 & 0.0822 \\ -0.2251 & 0.0850 & -0.1163 & -0.1163 & 0.0850 & -0.2251 \\ -0.2251 & 0.0850 & -0.1163 & -0.1163 & 0.0850 & -0.2251 \\ -0.2251 & 0.0850 & -0.1163 & -0.1163 & 0.0850 & -0.2251 \\ -0.2251 & 0.0850 & -0.1163 & -0.1163 & 0.0850 & -0.2251\end{array}\right]$

www.ijacsa.thesai.org 
The incidence matrix of the Biochemical ontology = flag $=0 ;$ incidate that the eigenvalues converged.

$\left[\begin{array}{llllllllllll}1 & 1 & 1 & 0 & 0 & 0 & 0 & 0 & 0 & 0 & 0 & 0 \\ 0 & 0 & 0 & 0 & 0 & 0 & 0 & 0 & 0 & 0 & 0 & 0 \\ 0 & 0 & 0 & 0 & 0 & 0 & 0 & 0 & 0 & 0 & 0 & 0 \\ 0 & 0 & 0 & 1 & 1 & 1 & 1 & 0 & 1 & 1 & 1 & 1 \\ 0 & 0 & 0 & 0 & 0 & 0 & 0 & 0 & 0 & 0 & 0 & 0 \\ 0 & 0 & 0 & 0 & 0 & 0 & 0 & 0 & 0 & 0 & 0 & 0 \\ 0 & 0 & 0 & 0 & 0 & 0 & 0 & 0 & 0 & 0 & 0 & 0 \\ 0 & 0 & 0 & 0 & 0 & 0 & 0 & 1 & 0 & 0 & 0 & 0 \\ 0 & 0 & 0 & 0 & 0 & 0 & 0 & 0 & 0 & 0 & 0 & 0 \\ 0 & 0 & 0 & 0 & 0 & 0 & 0 & 0 & 0 & 0 & 0 & 0 \\ 0 & 0 & 0 & 0 & 0 & 0 & 0 & 0 & 0 & 0 & 0 & 0 \\ 0 & 0 & 0 & 0 & 0 & 0 & 0 & 0 & 0 & 0 & 0 & 0 \\ 0 & 0 & 0 & 0 & 0 & 0 & 0 & 0 & 0 & 0 & 0 & 0\end{array}\right]$

Structural Dimension
Absolute Depth $=34$
Absolute Breadth $=18$
Density $=0.11$
Average Depth $=2.43$
Average Breadth $=3.6$
Maximal Depth $=4$
Maximal Breadth $=8$

The Transport Ontology

Transport ontologies aim to capture and represent different entities and the relations that exist between them in an accurate

and precise manner. Figures 5 described the class hierarchical structure of the transport ontology while figure 6 shows its corresponding graph in the protégé implementation.

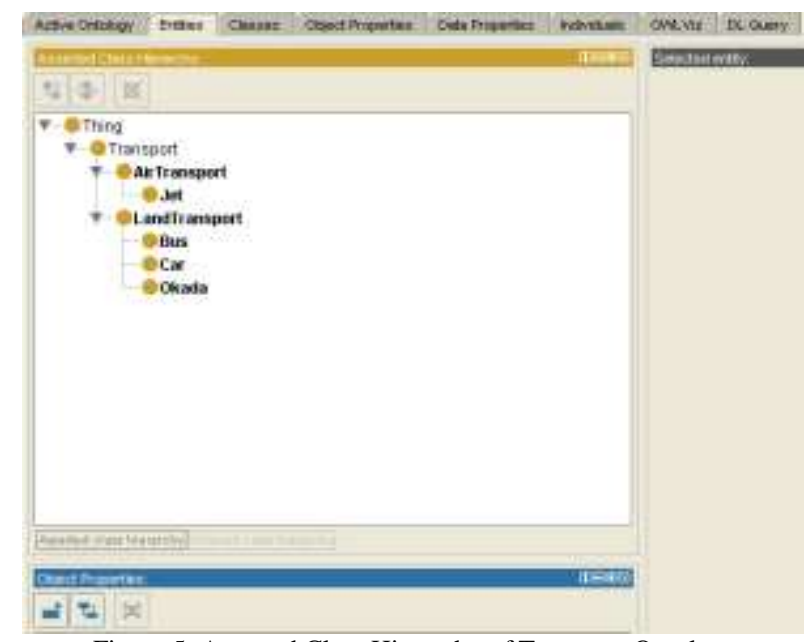

Figure 5. Asserted Class Hierarchy of Transport Ontology 


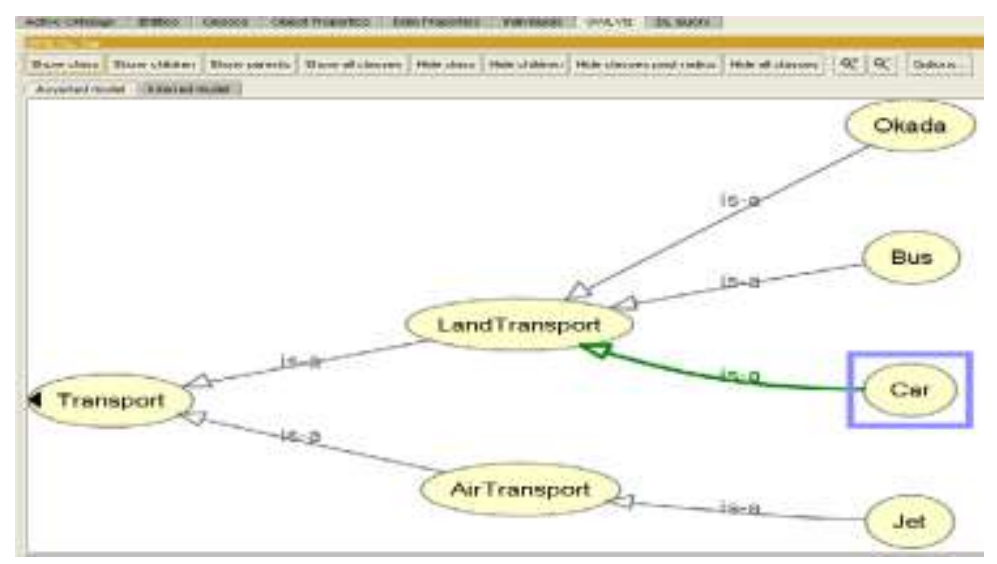

Figure 6. Transport OWL graph

Adjacency matrix (Transport) $=\left[\begin{array}{ccccccc}0 & 1 & 0 & 1 & 0 & 0 & 0 \\ 1 & 0 & 1 & 0 & 0 & 0 & 0 \\ 0 & 1 & 0 & 0 & 0 & 0 & 0 \\ 1 & 0 & 0 & 0 & 1 & 1 & 1 \\ 0 & 0 & 0 & 1 & 0 & 0 & 0 \\ 0 & 0 & 0 & 1 & 0 & 0 & 0 \\ 0 & 0 & 0 & 1 & 0 & 0 & 0\end{array}\right]$

The characteristics polynomial det $(A-\lambda I)=\lambda^{7}-6 \lambda^{5}+7 \lambda^{3}$

The Eigenvalues $=-2.1010,-1.2593,0,0,0.0000,1.2593,2.1010$

The Eigenvectors (V) $=\quad\left[\begin{array}{cccccc}-0.4397 & 0.3039 & -0.4377 & 0.4047 & 0.3039 & -0.4397 \\ -0.2706 & 0.6533 & 0.0000 & 0.0000 & -0.6533 & 0.2706 \\ -0.1288 & 0.5188 & 0.4377 & -0.4047 & 0.5188 & -0.1288 \\ -0.6533 & -0.2706 & 0.0000 & 0.0000 & 0.2706 & 0.6533 \\ -0.3109 & -0.2149 & 0.7437 & 0.2323 & -0.2149 & -0.3109 \\ -0.3109 & -0.2149 & -0.2452 & -0.7743 & -0.2149 & -0.3109 \\ -0.3109 & -0.2149 & -0.0608 & 0.1372 & -0.2149 & -0.3109\end{array}\right]$

The incidence matrix $=\left[\begin{array}{cccccc}1 & 0 & 1 & 0 & 0 & 0 \\ 0 & 1 & 0 & 0 & 0 & 0 \\ 0 & 0 & 0 & 0 & 0 & 0 \\ 0 & 0 & 0 & 1 & 1 & 1 \\ 0 & 0 & 0 & 0 & 0 & 0 \\ 0 & 0 & 0 & 0 & 0 & 0 \\ 0 & 0 & 0 & 0 & 0 & 0\end{array}\right]$

Structural Dimension
Absolute Depth $=8$
Absolute Breadth $=7$
Density $=0.29$
Average Depth $=4$
Average Breadth $=2.33$
Maximal Depth $=2$
Maximal Breadth $=4$ 


\section{CONCLUSION}

The work used the simplicity of an adjacency matrix of a graph to compute the algebraic spectrum and structural dimensions of ontology. It presented an application of the state of the art on ontology evaluation. The applications showed that the choice of suitable method must depend on the theme of ontology evaluation.

\section{REFERENCES}

[1] N. Alon and V. D. Isoperimetric Inequalities for Graphs and Super Concentrators, Journal of Combinatorial Theory, Series B 38, pp 73-88 0095-8956/85, Academic Press Inc., ( 1985)

[2] T. F. Coleman and J. J. More, Estimation of Sparse Jacobian Matrices and Graph Coloring Problems, SIAM journal on Numerical Analysis, vol 20(1), pp 187-209, (1983) Aussenac-Gilles and D. Sorgel, Supervised Text Analysis for Ontology and Terminology Engineering, Applied Ontology: An

[3] A. Gangemi, Ontology Design Patterns for Semantic Web Content, Proceedings of the fourth International Semantic Web Conference. Berlin Springer, (2005)

[4] Gangemi A., C. Catenacci, M. Ciaramita, J. Lehman, Modelling Ontology Evaluation, In Proceedings of the Third European Semantic Web Conference Berlin Springer, (2006)

[5] L. Lovasz, On the Shannon Capacity of a Graph, IEEE Trans. Inform. Theory, IT 25, pp 1-7, (1979)
[6] Q. Lu and V. Haarslev, OntoKBEval, DL-Based Evaluation of OWL Ontologies, In Proceedings of OWLED, (2006)

[7] B. Mohar, Some Applications of Laplace Eigenvalues of Graphs, Algebraic Methods and Applications, Eds G. Hahn serie C 497, pp 225 250, Kluwer, (1997)

[8] J. F. Sowa, Ontology, http://www.jfsowa.com/ontology

[9] S. Staab, Handbook on Ontologies, Springer (2009)

[10] A. Maedche and S. Staab, Measuring Similarity between Ontology, Proceedings CIKM LNAI vol. 2473, (2002)

[11] P. Spyn, Assessing Triples Mined from Texts, Technical Report 09, STAR Lab, Brussel Belgum, (2005)

[12] R. Porzel and R. Malaka, A Task Based Approach for Ontology Evaluation, ECA/2004, Workshop on Ontology Learning and population (2004)

[13] P. Avesani, F. Giunchiglia, and M. Yatskevish, A large Scale Taxonomy Mapping Evaluation, In Proceedings of the International Semantic Web Conference ISUC, (2005)

[14] D. Hull, Evaluating Evaluation Measures Stability, In Proceedings of SIGIR (2000)

[15] A. Lozano-Tello and A. Gomez-Perez, Ontometric: A Method to Choose the Appropriate Ontology, Journal of Database Management Vol 15(2), pp 1-18,(2004)

[16] A. Gomez-Perez, A Toward a Framework to verify Knowledge Sharing Technology: Expert Systems with Applications Vol. 11(4), pp 519-529, (1996)

[17] K. Superkar, A Peer-Review Approach for Ontologgy Evaluation, Proceedings $8^{\text {th }}$ International Protégé Conference, Madrid, Spain (2005) 